\title{
Relações entre actividade física, aptidão física, morfológica e coordenativa na infância e adolescência
}

\author{
Relationships between physical activity, physical fitness, somatic fitness, \\ and coordination along childhood and adolescence
}

\author{
J.P. Saraiva, L.P. Rodrigues
}

RESUMO

Neste trabalho procuramos perceber como se estabelecem as relações entre a actividade física, a aptidão física, a aptidão morfológica e a aptidão coordenativa ao longo do percurso de desenvolvimento motor de crianças e jovens, e qual o possível impacto sobre a sua vida futura. Foi realizada uma pesquisa na base de dados B-On e seleccionados os trabalhos publicados na última década (2000 a 2009) e que estudaram as relações entre qualquer um dos factores de interesse. No seu conjunto, e não obstante as diferenças metodológicas ao nível dos instrumentos utilizados na recolha dos dados, os artigos revistos sugerem a existência clara de associações positivas entre todos os factores estudados, mas com especial relevância para os resultados relativos à actividade física e a aptidão coordenativa. É ainda perceptível um interesse actual e particular dos investigadores relativamente à actividade física e aos níveis de coordenação motora exibidos por crianças e jovens, bem como aos seus resultados na determinação das condições de saúde ao longo da vida.

Palavras-chave: actividade física, aptidão física, aptidão morfológica, aptidão coordenativa, desenvolvimento motor

\section{ABSTRACT}

The two main goals of this review were to understand how the relationships between physical activity, physical fitness, somatic fitness, and coordination are established along the motor development of children and adolescents, and how they would influence their future lives. The web based bibliographic database B-On was searched for peer-reviewed publications during the last decade (2000 to 2009). Search criteria included all articles on relationships between any two of the above named factors. Although different methodological designs and variables were found as markers for the same factor, overall results suggested the existence of a clear positive relationship among physical activity, physical fitness, somatic fitness, and coordination from childhood to adolescence, with a special relevance for the relationship between physical activity and coordination. It was also noted a renewed interest on physical activity and motor coordination developmental characteristics and relationships as well as on their lifelong health effects.

Keywords: physical activity, physical fitness, somatic fitness, motor coordination, motor development

Submetido: 08.09.2009 | Aceite: 02.05.2010

João Paulo Saraiva. Centro de Investigação em Literacia e Bem-Estar da Criança, Universidade do Minho Portugal.

Luís Paulo Rodrigues. Centro de Investigação em Desporto, Saúde e Desenvolvimento Humano; Instituto Politécnico de Viana do Castelo - Portugal.

Endereço para correspondência: Luís Paulo Rodrigues, Escola Superior de Educação, Instituto Politécnico de Viana do Castelo, Av. Capitão Gaspar de Castro, Apartado 513, 4901-908 Viana do Castelo - Portugal.

E-mail: lprodrigues@ese.ipvc.pt 
Crescimento e morfologia, aptidão física e coordenativa e envolvimento em actividades físicas serão sem dúvida alguns dos mais estudados assuntos no desenvolvimento motor infanto-juvenil. A forma como os investigadores os têm abordado configura uma multiplicidade de factores e variáveis de medida que, por parcimónia, convém interpretar de forma conjunta. A Aptidão Morfológica (ApM), entendida como as características morfológicas associadas ao nível de prontidão motora e robustez músculoesquelética do indivíduo, tem sido observada através de indicadores dimensionais simples (estatura, peso, perímetros musculares, e pregas adiposas) e complexos [índice de massa corporal (IMC), percentagem de massa gorda (\%MG), somatótipo, etc.], levando-nos a conhecer bem os valores normativos destes indicadores ao longo do ciclo de vida e a sua influência na determinação de uma vida saudável (e.g. Carter, 1988; Cole, Bellizzi, Flegal, \& Dietz, 2000; McDowell, Fryar, Hirsch, \& Ogden, 2005; Padez, Fernandes, Mourão, Moreira, \& Rosado, 2004; Rodrigues, Sá, Bezerra, \& Saraiva, 2006). O conceito de Aptidão Física (ApF) dispensa apresentações e tem sido largamente monitorizado ao longo do último século (e.g. Reiff et al., 1986; Simon et al., 1990), parecendo representar um elemento fundamental para o sucesso motor, propiciando êxito na actividade e criando expectativas de manutenção futura de estilos de vida activos (Andersen, Hasseltrom, Gronfeldt, Hansen, \& Karsten, 2004; Janz, Dawson, \& Mahoney, 2000; Malina, 2001). A preocupação com a Aptidão Coordenativa (ApC), marcadora da proeficiência motora, tem vindo a evidenciar-se na literatura dos últimos anos com a adopção de instrumentos estandardizados e validados para a avaliação da qualidade do comportamento motor de crianças e jovens [e.g. Test of Gross Motor Development 2 (TGMD-2), Movement Assessment Battery for Children (M-ABC), Peabody Developmental Motor Scale 2 (PDMS-2), Köperkoordinations-test für Kinder (KTK)] e a sugestão de que os níveis de ApC poderão influenciar decisivamente a forma como as crianças se motivam e envolvem em práticas motoras (Okely, Booth, \& Patterson, 2001). Por último, a Actividade Física (AF) tem sido descrita como um dos mediadores (senão o mediador) mais importante na determinação epidemiológica da saúde das populações (Blair \& Church, 2004), originando uma preocupação especial no estudo e caracterização dos episódios de AF das populações, particularmente nas faixas etárias mais novas onde a modelação dos comportamentos pode pressupor a criação de hábitos para a vida.

Da constatação da importância destes factores emerge a necessidade de compreender o modo efectivo como interagem na infância e juventude, desencadeando a oportunidade deste trabalho. Nele procuraremos referenciar as investigações desenvolvidas em diferentes contextos geográficos e culturais, mas que corroboram da mesma preocupação em perceber a interacção destes factores no desenvolvimento motor presente e futuro das populações.

\section{MÉTODO}

$\mathrm{Na}$ pesquisa dos estudos publicados recorremos à $\mathrm{B}-\mathrm{O}$, base bibliográfica online que junta diversos recursos de pesquisa (PubMed, Search Direct, Web of Science, Scielo, etc). Os critérios de procura seleccionados permitiram-nos consultar os trabalhos publicados na última década (2000 a 2009), através do cruzamento de palavras-chave especificamente relacionadas com o objectivo da revisão (Physical Activity, Physical Fitness, Motor Skills, Motor Coordination, Motor Competence, Growth, Weight Status, Obesity). No conjunto dos trabalhos revistos, diferentes variáveis são definidas dentro de cada um dos factores associados ao Desenvolvimento Motor (DM). Assim, para a ApM as mais usadas foram as medidas antropométricas básicas (peso e altura), o índice de massa corporal e as pregas adiposas. O nível de proficiência nas habilidades motoras fundamentais e o nível de 
desempenho em testes específicos de DM marcam as formas mais usuais de avaliação da ApC. Relativamente à $\mathrm{ApF}$ foram utilizados testes de resistência aeróbica, de força (superior, média e inferior), flexibilidade e velocidade. Por fim, para a AF, as medidas utilizadas procuraram avaliar a frequência, a duração e/ou a intensidade dos momentos de prática através de medições directas (acelerómetros e pedómetros) ou indirectas (questionários). A existência de resultados relativos à relação entre pelo menos dois dos factores pesquisados durante o período da infância ou adolescência constituiu critério de inclusão para os estudos aqui apresentados.

Quanto aos delineamentos experimentais utilizados no esclarecimento desta questão, encontramos sobretudos estudos correlacionais que visam inferir sobre a relação entre as variáveis em estudo e recorrem a métodos longitudinais e transversais, sendo que neste último caso se torna difícil controlar outras potenciais influências, nomeadamente $\mathrm{O}$ estatuto socioeconómico da amostra envolvida (Willms, 2003). De todos os trabalhos referenciados nesta revisão, em apenas um foi implementado um programa de intervenção onde foram deliberadamente controlados os factores com influência directa nas variáveis em estudo.

Seguindo esta linha orientadora, estruturamos a apresentação desta revisão descrevendo o ano, o local (país), as características da amostra, o delineamento experimental e os resultados obtidos em cada estudo. Para ajudar o leitor na leitura e comparação, foi mantida a ordem cronológica das publicações e elaborada uma tabela síntese de todos os estudos revistos.

\section{REVISÃO DA LITERATURA}

Grund et al. (2000), ao estudarem a relação entre a AF (questionário e frequência cardíaca), ApF (ergoespirometria e tensiometria computadorizada dos membros inferiores) e a ApM [peso, altura, IMC e Massa Gorda (MG)] de 88 crianças alemãs dos 5 aos
11 anos de idade, observaram que as crianças mais altas eram tendencialmente mais activas e que crianças com baixo nível de aptidão física eram mais pesadas e apresentavam valores mais elevados de IMC e MG. Aliás, também Oja (2001), no seu artigo de revisão relativamente à $\mathrm{AF}$ e seus efeitos na $\mathrm{ApF}$ e estado de saúde, refere uma forte evidência da relação entre o volume semanal de $\mathrm{AF}$ e a aptidão cardiorespiratória (ApF). No entanto, aponta o mesmo autor que apenas foram encontrados fracos indícios de uma relação entre o volume total de AF e outras medidas relativas ao estado de saúde.

Integrado no Amsterdam Growth and Health Study (AGHS), Minck, Ruiter, Van Mechelen, Kemper e Twisk (2000), seguiram longitudinalmente 83 rapazes e 98 raparigas entre os 13 e os 27 anos de idade, avaliados em seis momentos nas suas características de ApF [Motor Performance (MOPER) Fitness Test], de AF (questionário), e ApM (somatório pregas adiposas). A relação longitudinal encontrada entre estas variáveis permitiu concluir pela existência de uma associação significativa entre a adiposidade (ApM) e a velocidade $(r=-.28$, $r=-.31$, respectivamente para rapazes e raparigas), a força inferior $(r=-.38, r=-.32)$, a força média $(r=-.23, r=-.18)$, e a resistência aeróbica $(r=-.40, r=-.44)$, ao longo deste período do ciclo de vida. Já a $\mathrm{AF}$ apenas demonstrou associar-se de forma significativa, embora fraca, com a resistência aeróbica $(r=.17, r=.10)$.

No ano seguinte, Kemper, Vente, Mechelen, e Twisk (2001) reportaram os resultados do mesmo estudo (AGHS), mas agora relativamente ao percurso longitudinal de cerca de 400 sujeitos desde os 13 até aos 33 anos, e com o objectivo de estabelecer a relação entre a ApF (MOPER Fitness Test) na adolescência e a AF (inquérito) na idade adulta. Os resultados demonstraram que, dos testes de aptidão física realizados na adolescência, apenas a prova de resistência (12 minutos em corrida) e o consumo máximo de oxigénio $\left(\mathrm{VO}_{2} \max \right)$ se revelaram como predictores do nível de 
actividade física na idade adulta. Os autores acrescentam ainda que estas constatações foram mais significativas nas raparigas que nos rapazes. No mesmo ano, Ball et al. (2001) investigaram a relação entre a $\mathrm{AF}$ [Double Labeled Water (DLW)] e a ApM (IMC, MG e \%MG) de 106 crianças australianas dos 6 aos 10 anos de idade e observaram a existência de uma correlação significativa em rapazes $(r=$ -.37 para o IMC, $r=-.46$ para a MG e $r=$ -.50 para $\% \mathrm{MG}$ ), e nula para raparigas.

Graf et al. (2004) testaram 668 crianças alemãs (6.7 anos de idade média) quanto à sua ApM (peso, altura e IMC), aos hábitos de AF (questionário), à $\mathrm{ApF}$ (corrida de $6 \mathrm{~min}$ ) e $\mathrm{ApC}$ (KTK), tendo verificado que a ApM se correlacionava significativamente, quer com a ApF, quer com a ApC (KTK e IMC, $r=-.16$, corrida 6-min e IMC, $r=-.20$ ). Foi também evidente neste estudo que as crianças obesas ou com excesso de peso obtiveram piores resultados ao nível da coordenação motora, e que as crianças mais activas eram significativamente mais aptas nas suas prestações coordenativas (ApC). Também Reed e Metzker (2004), ao examinarem a relação entre a AF diária (pedometria) e em aulas de Educação Física (EF) e a ApC (Bass Stick Balance Test; Side-Step Agility Test e AAHPERD Passing Test) de 217 crianças norteamericanas dos $6^{\circ}, 7^{\circ}$ e $8^{\circ}$ anos de escolaridade, observaram correlações totais significativas entre o AAHPERD Passing Test e os níveis de AF diários $(r=.35)$, e entre o SideStep Agility Test e o número de passos nas sessões de EF $(r=.27)$.

Fisher et al. (2004), ao estudarem a relação entre a AF (acelerometria) e o desempenho nas habilidades motoras fundamentais (M-ABC), de 394 crianças escocesas em idade pré-escolar, encontraram uma tendência para as crianças que despendiam mais tempo em actividade física moderada ou vigorosa (AFMV) apresentarem melhor proficiência no desempenho das habilidades motoras. Os valores da correlação entre o desempenho motor global (ApC) com a AF total $(r=.10)$ e o tempo dispendido em AFMV $(r=.18)$ foram positivos e significativos em ambos os sexos. Também Dencker et al. (2006), ao avaliarem a AF diária (acelerometria) e a $\mathrm{ApF}\left(\mathrm{VO}_{2} \max \right.$ avaliado em cicloergómetro) de 248 crianças suecas dos 8 aos 11 anos de idade, encontraram associações positivas e significativas entre a AF total e o $\mathrm{VO}_{2} \max (r=$ .23 para rapazes e raparigas), mas uma relação mais forte foi observada quando o nível de ApF foi interpretado em função do tempo gasto na prática de AFMV ( $r=.32$ para rapazes, $r=.30$ para raparigas).

Na mesma linha de resultados, Wrotniak, Epstein, Dorn, Jones e Kondilis (2006), ao examinarem a relação entre a ApC (BruininksOseretsky Test of Motor Proficiency), a AF (acelerometria), e a ApM (IMC) de 65 crianças norte-americanas dos 8 aos 10 anos de idade, verificaram que a proficiência motora das crianças encontrava-se positivamente correlacionada com a AF total $(r=.32)$ e a percentagem de tempo dispendida em AFMV $(r=.30)$, e inversamente correlacionada com o tempo passado em actividades sedentárias ( $r=$ -.31). Por outro lado, crianças menos aptas morfologicamente (maior IMC) demonstraram serem menos activas e possuírem pior desempenho motor que os seus pares mais aptos (com menor IMC).

Em Portugal, Mota, Flores, Flores, Ribeiro e Santos (2006) avaliaram 127 rapazes e 128 raparigas com 8 e 9 anos de idade, relativamente à $\mathrm{ApF}$ (corrida milha) e à $\mathrm{ApM}$ (IMC), tendo encontrado uma correlação significativa entre estes dois parâmetros $(r=$ $-.36)$, apenas nas raparigas.

Ao avaliarem a ApC (Bruininks-Oseretsky Test of Motor Proficiency), a ApF (20-m Shuttle Run Test) e a AF (Children's Self-Perceptions of Adequacy in and Predilection for Physical Activity) de 586 crianças canadianas do $4 .^{\circ}$ ao $8 .^{\circ}$ ano de escolaridade, Cairney, Hay, Wade, Faught e Flouris (2006) verificaram que crianças a quem foram diagnosticados distúrbios de desenvolvimento da coordenação motora revelaram um baixo nível de aptidão física e 
pouco ou nenhum envolvimento em actividades físicas devido a uma autopercepção negativa da sua competência motora para a prática das mesmas.

$\mathrm{Na}$ Estónia, Raudsepp e Päll (2006) encontraram uma associação entre o nível das habilidades motoras fundamentais $(r=.44$ para o lançar e $r=.55$ para o saltar) de 133 crianças (7.6 anos de idade média) e a sua participação em actividades físicas realizadas fora do ambiente escolar e que exigem um domínio motor especializado (skill-specific). No entanto, o mesmo não aconteceu quando se considerou a AF total destas crianças, o que segundo os autores talvez se deva ao facto deste tipo de habilidades estarem especificamente associadas a episódios de AF normalmente realizados em contexto específico desportivo de treino e competição.

Ara, Moreno, Leiva, Gutin e Casajús (2007) investigaram o possível contributo da AF (avaliada pela participação em actividades físicas extra-curriculares) na ApM (somatório de pregas adiposas e IMC) e na ApF (bateria EUROFIT) de 1068 crianças espanholas dos 7 aos 12 anos de idade. Os resultados obtidos revelaram que as raparigas sedentárias tinham maior adiposidade subcutânea do que as suas parceiras mais activas $(p<.05)$, que a AF produzia um efeito positivo e significativo na ApM dos rapazes e que a aptidão aeróbica $\left(\mathrm{VO}_{2}\right.$ max $)$ se encontrava negativamente relacionada com a adiposidade em ambos os sexos.

Estes resultados estão também de acordo com os encontrados por Stratton et al. (2007), que seguiram 15621 crianças inglesas dos 9 aos 11 anos de idade entre 1998 e 2004, e observaram um declínio anual concomitante nos níveis de ApF (20-m Shuttle Run Test) e na ApM (aumento do IMC), sugerindo uma relação entre ambos.

Nos Estados Unidos da América, uma amostra de 230 crianças entre os 7 e os 12 anos de idade, representativa da zona de Illinois, foi avaliada na sua ApF (Fitnessgram),
ApM (IMC), AF (questionários, entrevista e pedometria) e ApC (teste de habilidades motoras/desportivas) (Castelli \& Valley, 2007). Os resultados encontrados pelos autores mostram uma baixa, mas significativa associação entre a ApM e os testes de ApF ( $r=$ -.24 a -.29 ); correlações moderadas entre a $\mathrm{ApC}$ e a $\mathrm{ApF}(r=.57$ para a resistência aeróbica, $r=.39$ para a força média e $r=.36$ para a força superior). Por sua vez a AF diária demonstrou encontrar-se relacionada com a ApC $(r=.55)$ e com os testes de ApF $(r=.28$ a .46).

Por sua vez, Lazaar et al. (2007), num programa experimental de intervenção em $\mathrm{AF}$ desenvolvido durante seis meses com 425 crianças francesas dos 6 aos 10 anos de idade em contexto escolar, relataram que no conjunto dos dois sexos se verificaram alterações favoráveis e significativas em todos os índices de ApM avaliados (perímetro da cintura, pregas adiposas, massa magra nas raparigas, e IMC e massa magra nos rapazes) relativamente ao grupo de controlo. Estas conclusões, segundo os autores, estabelecem uma relação evidente entre a $\mathrm{AF}$ e a $\mathrm{ApM}$, sugerindo que a AF pode ser utilizada para prevenir e corrigir problemas de excesso de peso e obesidade nesta faixa etária, sobretudo no sexo feminino.

Procurando determinar possíveis relações entre a ApF (Test of Physical Fitness) e a ApC (M-ABC), Haga (2008) avaliou 67 crianças norueguesas, com 9 e 10 anos de idade. Nos seus resultados esta autora reporta correlações significativamente fortes e moderadas entre os valores globais da ApF e da ApC ( $r=-.59)$; e entre os valores globais da $\mathrm{ApF}$ e parciais da ApC $(r=-.44, r=-.38$ e $r=-.49$; respectivamente para a destreza manual, as habilidades com bola e o equilíbrio). Analisando as relações por géneros, percebe-se que esta associação é sempre mais forte nas raparigas $(r=-.40$ a -.78$)$, comparativamente com os rapazes $(r=-.22 \mathrm{a}-.53)$. 
Quadro 1

Relação dos estudos revistos por ordem cronológica

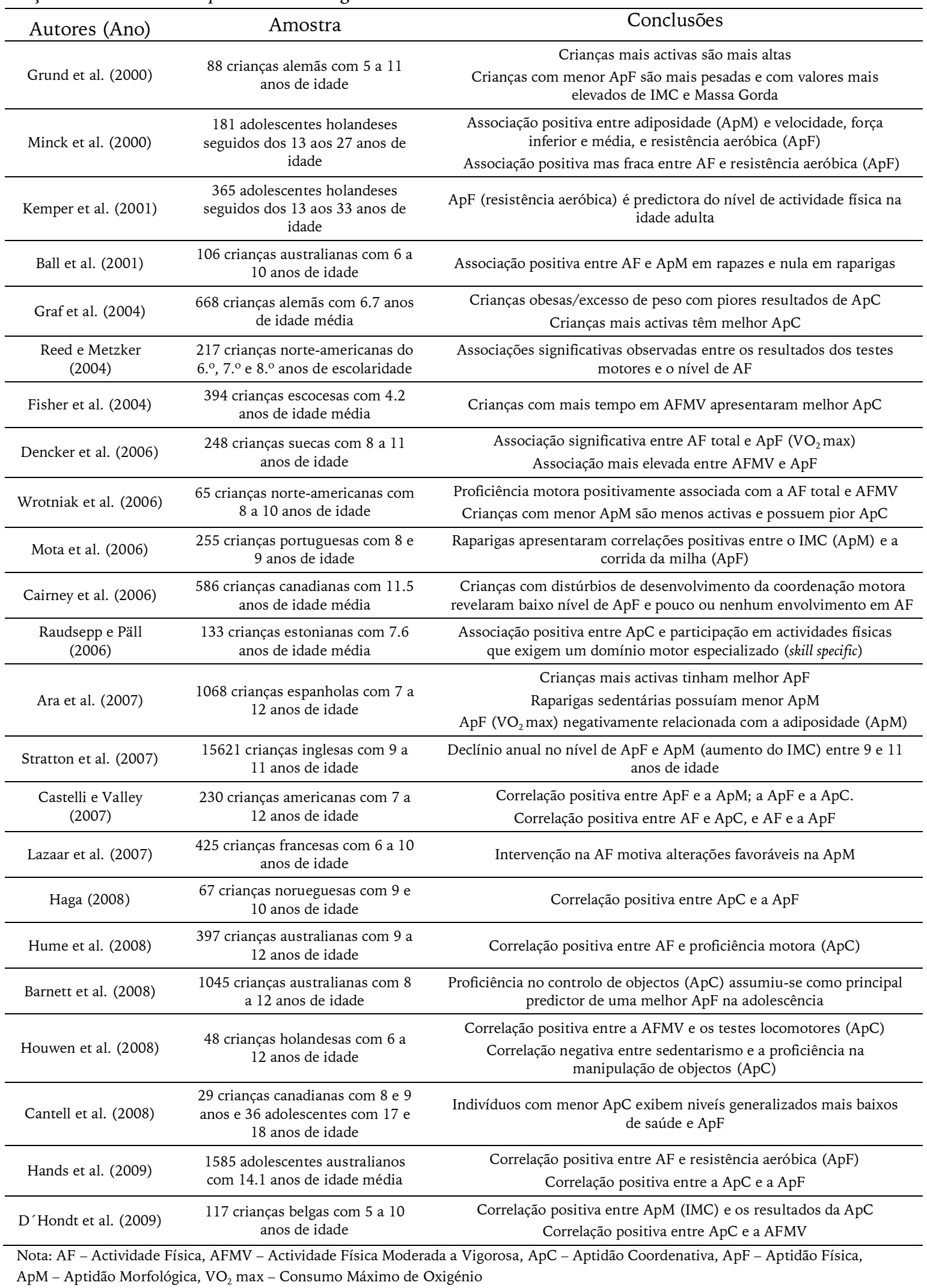


Relativamente à associação entre a $\mathrm{ApC}$ e a AF, Hume et al. (2008) indagaram sobre a proficiência na execução de seis habilidades motoras fundamentais (ApC) e a AF (acelerometria) de 397 crianças australianas dos 9 aos 12 anos de idade. Nos seus resultados relatam a existência de uma correlação positiva e significativa entre a AF e o nível de proficiência motora nas habilidades motoras $(r=.25$ para rapazes e $r=.21$ para raparigas). Os autores acrescentam que nos rapazes as actividades de intensidade moderada $(r=.24)$ e moderada/vigorosa $(r=$ .24) estavam associadas positivamente às tarefas de manipulação de objectos, enquanto que apenas as actividades de intensidade vigorosa $(r=.22)$ foram responsáveis pela melhor proficiência nas tarefas de locomoção. No caso das raparigas, apenas as actividades vigorosas estiveram significativamente correlacionadas com as tarefas de locomoção $(r$ $=.29)$. $\mathrm{Na}$ mesma linha de conclusões, Barnett, Beurden, Morgan, Books e Beard (2008), num estudo sobre a relação entre a proficiência nas habilidades motoras fundamentais $(\mathrm{ApC})$ de 276 crianças australianas aos dez anos de idade e a ApF (20$m$ Shuttle Run Test) seis anos depois, concluíram que a proficiência nas habilidades de controlo de objectos na infância assumiu o principal efeito como predictor de uma aptidão aeróbica favorável durante a adolescência.

Houwen, Hartman e Visscher (2008), ao estudarem a relação entre a $\mathrm{AF}$ (acelerometria), ApM (peso, altura e IMC) e a ApC (TGMD-2) de 48 crianças holandesas dos 6 aos 12 anos de idade, constataram uma ausência de associações significativas entre os parâmetros de avaliação da ApM e AF em estudo. No entanto, correlações significativas foram observadas entre a percentagem de tempo dedicado à realização de AFMV e os resultados dos testes locomotores $(r=.38)$, sugerindo que o nível de proficiência motora condicionou o envolvimento das crianças em actividades físicas de intensidades superiores. Correlações inversas e significativas também foram observadas entre as actividades sedentárias e a proficiência na manipulação de objectos $(r=-.31)$, o que sugere que crianças com os melhores resultados neste tipo de tarefas motoras dedicavam menos tempo às actividades menos activas comparativamente com o restante da amostra.

Cantell, Crawford e Doyle-Baker (2008) procuraram relacionar o nível de competência motora (ApC), a ApF, a ApM (IMC e perímetro da cintura) e indicadores metabólicos de saúde, em 149 crianças, adolescentes e adultos canadianos. Nas suas observações, os autores reportam que o nível de $\mathrm{ApC}$ assumiu um papel preponderante nos resultados dos testes de ApF, ApM e saúde realizados em todas as idades. Indivíduos com menor competência motora tiveram valores de IMC significativamente mais elevados que os seus pares mais coordenados e as crianças com maior competência motora foram capazes de níveis de actividade física mais intensos. $\mathrm{Na}$ generalidade os autores concluem que indivíduos com menor competência motora exibem níveis generalizados mais baixos de saúde e ApF.

Recentemente, D'Hondt, Deforche, Bourdeaudhuij e Lenoir (2009), num estudo sobre a relação entre a ApC (M-ABC), a ApM (peso, altura e IMC) e a AF (acelerometria) de 117 crianças belgas dos 5 aos 10 anos de idade, observaram que o IMC se revelou significativamente associado aos resultados dos testes motores $(r=-.34)$. No entanto, não houve $o$ registo de diferenças motoras significativas entre crianças classificadas com peso normal e excesso de peso, o que segundo os autores, parece sugerir a existência de um ponto de corte de adiposidade a partir do qual emergem as dificuldades de coordenação. Quanto à AF, com excepção da prova de destreza manual, os demais resultados apontam para uma correlação significativa entre ApC e a prática de AFMV $(r=-.21$ a -.25 , sendo que no teste $\mathrm{M}-\mathrm{ABC}$ os melhores resultados correspondem aos valores mais baixos), que pode ser entendida como um 
reflexo de um estilo de vida inactivo normalmente adoptado por crianças tendencialmente obesas ou obesas de facto.

Examinando a associação entre a $\mathrm{AF}$ (pedometria), ApF (Australian Fitness Education Award), e a ApM (IMC) e a ApC (McCarron Assessment of Neuromuscular Development), Hands, Larkin, Parker, Straker e Perry (2009) testaram 1585 adolescentes (771 raparigas e 814 rapazes) com 14.1 anos de média de idade. A AF apenas demonstrou associação significativa com a prova de resistência aeróbica $(r=.16$ e $r$ $=.23$, respectivamente em rapazes e raparigas). As correlações entre a ApC e a ApF foram significativas nos dois sexos, mas ligeiramente mais elevadas nos rapazes $(r=$ .22 a .43 nos rapazes e $r=.15$ a .33 nas raparigas). A ApM demonstrou fraca ou nenhuma correlação com todos os outros factores.

\section{CONSIDERAÇÕES FINAIS}

Ao longo desta revisão procuramos dar um panorama dos resultados da investigação da última década que nos podem permitir melhor compreender as características das associações existentes entre factores que indubitavelmente condicionam o sucesso motor ao longo do desenvolvimento da criança e do jovem. Uma limitação óbvia na interpretação destes resultados deriva da impossibilidade de comparação directa dos mesmos, dada a grande diversidade de aproximações metodológicas, de instrumentos e técnicas utilizadas na avaliação das variáveis, e da escolha de medidas de associação reportadas. Ainda assim, os estudos revistos apontam para a existência clara de associações positivas entre os factores estudados, ao longo do desenvolvimento infanto-juvenil: nomeadamente entre a ApM e a AF (3 estudos), entre a ApF e a ApM (4 estudos), entre a ApM e a AF (5 estudos), entre a ApF e a AF (6 estudos), entre a ApF e a ApC (7 estudos) e entre a AF e a ApC (10 estudos).

Se, para evitar a dispersão resultante da diversidade metodológica, centrarmos a nossa atenção exclusivamente nos estudos que reportam medidas típicas de associação (coeficientes de correlação) entre qualquer um dos factores revistos, percebemos que a relação entre o envolvimento em $\mathrm{AF}$ e as diversas facetas que expressam o nível de $\mathrm{ApF}$ de crianças e adolescentes é clara $(r=.10$ a .46$)$ (Castelly \& Valley, 2007; Dencker et al., 2006; Hands et al., 2009), mas que esta relação é principalmente favorecida quando as crianças se envolvem em actividades mais vigorosas (Dencker et al., 2006). Relativamente à associação entre a AF e uma melhor ApM (menor \%MG), apenas foram encontradas valores significativos em crianças do sexo masculino ( $r=.50)$ (Ball et al., 2001). A competência motora é o factor que mais se parece associar com a AF de crianças em idade escolar $(r=.21$ a .55$)$ e pré-escolar $(r=.18)$ (Castelly \& Valley, 2007; D'Hondt et al., 2009; Fischer et al., 2004; Houwen et al., 2008; Hume et al., 2008; Raudsepp \& Päll, 2006; Reed \& Metzker, 2004; Wrotniak et al., 2006).

Relativamente à $\mathrm{ApF}$, e para além da associação com a AF já descrita, a relação com a ApM foi estabelecida de forma moderada $(r$ $=.24$ a .29) para crianças de ambos os sexos (Castelli \& Valley, 2007) e com a resistência aeróbica apenas para as raparigas $(r=.36)$ (Mota et al., 2006). Curiosamente, em adolescentes esta associação não se revelou significativa (Hands et al., 2009). Por sua vez, a competência motora (ApC) aparece como o factor que incontestavelmente mais se relaciona com a ApF de crianças $(r=.30$ a .59) e adolescentes $(r=.15$ a .43$)$ (Castelli \& Valley, 2007; Haga, 2008; Hands et al., 2009).

Por último, os resultados revistos sugerem que a relação entre a ApM e a ApC acontece de forma evidente, mas moderada, em crianças de ambos os sexos ( $r=.16$ a .34) (D'Hondt et al., 2009; Graf et al., 2004)

No que diz respeito ao eventual sentido dos efeitos, a conclusão desta revisão é menos clara já que apenas três dos trabalhos foram delineados com esse objectivo. Num deles concluiu-se que a ApF (especificamente a 
resistência aeróbica) na infância foi predictora dos níveis de actividade física na idade adulta (Kemper et al., 2001). Num segundo, um programa de intervenção na $\mathrm{AF}$ resultou em modificações favoráveis na ApM (Lazaar et al., 2007). E, no último, a ApC (habilidades de controlo de objectos) na infância demonstrou poder preditivo na expressão da aptidão aeróbica (ApF) após a puberdade (Barnett et al., 2008).

Um outro aspecto que importa realçar é que a grande maioria (19) dos artigos aqui revistos se debruça sobre a $\mathrm{AF}$ e/ou a $\mathrm{ApC}$, sendo que dez abordam especificamente a relação entre estes dois factores. Este facto é elucidativo das preocupações dos investigadores na última década. O lugar de destaque da AF parece-nos óbvio, dada a apreensão actual da sociedade com o sedentarismo e sua expressão na saúde das populações. Já a avaliação do papel da ApC ao longo do percurso de desenvolvimento parece constituir uma re-centração dos investigadores numa característica do desenvolvimento motor que parecia vir a perder fulgor desde o final dos anos 80. A este ressurgimento não deverá ser estranho o aparecimento de um conjunto de investigadores e estudos a sugerir que a melhoria na proficiência motora das crianças pode ter o potencial de influenciar os níveis de AF habitual para lá da idade escolar, e portanto o potencial indispensável para influenciar as decisões políticas acerca da promoção da saúde (e.g. Butcher \& Eaton, 1989; Rose, Larkin, \& Berger, 1998; Stodden et al., 2008; Sallis, Prochaska, \& Taylor, 2000).

Apesar da concordância generalizada dos estudos, fica a impressão evidente da dificuldade na inquirição ecológica destas variáveis. Inúmeros factores relativos ao indivíduo, mas também às suas trajectórias de vida e ao meio envolvente parecem confundir as perguntas dos investigadores e poder contaminar as suas conclusões.

Perante esta complexidade experimental, a consistência dos resultados apresentados (mesmo que os valores de associação não sejam elevados) parecem demonstrar a existência inequívoca de fortes ligações desenvolvimentais entre a AF dos sujeitos, a sua $A p F$, o seu nível de proficiência no controlo das habilidades motoras (ApC), e as características morfológicas (ApM). Claro que seria esperado que todos e cada um dos factores aqui estudados demonstrassem agregação, associação evidente, já que todos eles contribuem de forma saliente para o sucesso motor das crianças e jovens. Pretendese que as crianças possam possuir uma boa aptidão morfológica e física, que sejam proficientes no domínio motor e que se mantenham activas. Espera-se também que uma menor expressão de qualquer um destes factores influencie a expressão de todos os outros.

Mas a pergunta que se impõe é se existirá uma forma melhor e mais simples de podermos influenciar o trajecto de desenvolvimento motor das crianças. Por outras palavras, se algum destes factores poderá assumir um papel preponderante para influenciar todos os outros, e com eles o sucesso motor das crianças com todas as possíveis consequências na sua vida futura. Qual a pedra de toque fundamental sobre a qual deveremos apontar os nossos recursos educacionais e sociais? Apesar de esta pergunta não poder ser cabalmente respondida por este conjunto de estudos, o facto de a ApC aparecer recorrentemente como $\mathrm{O}$ factor mais fortemente associado a qualquer um dos outros, parece sugerir a ideia que a competência motora na execução das habilidades motoras fundamentais deve constituir preocupação fundamental na educação formal e informal das crianças actuais.

Para esclarecer devidamente esta questão resta-nos esperar que a próxima década nos possa trazer mais e melhores esclarecimentos sobre os pormenores destas ligações. Sobretudo sobre o sentido e magnitude dos seus efeitos na infância, e ao longo do tempo de desenvolvimento. 


\section{REFERÊNCIAS}

Andersen, L., Hasseltrom, H., Gronfeldt, V., Hansen, S., \& Karsten, K. (2004). The relationship between physical fitness and clustered risk, and tracking of clustered risk from adolescence to young adulthood: Eight years follow-up in the Danish Youth and Sport Study. International Journal of Behavioral Nutrition and Physical Activity, 1, 6.

Ara, I., Moreno, L., Leiva, M., Gutin, B., \& Casajús, J. (2007). Adiposity, physical activity and physical fitness among children from Aragón, Spain. Obesity, 15, 1918-1924.

Ball, E., O'Connor, J., Abbott, R., Steinbeck, K., Davies, P., \& Wishart, C. (2001). Total energy expenditure, body fatness, and physical activity in children aged 6-9 y. American Journal of Clinical Nutrition, 74, 524-528.

Barnett, L., Beurden, E., Morgan, P., Books, L., \& Beard, J. (2008). Does childhood motor skill proficiency predict adolescent fitness? Medicine $\&$ Science in Sports \& Exercise, 40, 2137-2144.

Blair, S., \& Church, T. (2004). The fitness, obesity, and health equation: Is physical activity the common denominator? Journal of the American Medical Association, 292, 1232-1234.

Butcher, J., \& Eaton, W. (1989). Gross and fine motor proficiency in pre-schoolers: Relationships with free play behaviour and activity level. Journal of Human Movement Studies, $16,27-36$.

Cairney, J., Hay, J., Wade, T., Faught, B., \& Flouris, A. (2006). Developmental coordination disorder and aerobic fitness: Is it all their heads or is measurement still the problem? American Journal of Human Biology, 18, 66-70.

Cantell, M., Crawford, S., \& Doyle-Baker, P. (2008). Physical fitness and health indices in children, adolescents and adults with high or low motor competence. Human Movement Science, 27, 344362.

Carter, J. (1988). Somatotypes of children in sports. In R. Malina (Ed.), Young athletes. Biological, psychological and educational perspectives (pp. 153165). Champaign, IL: Human Kinetics.

Castelli, D., \& Valley, J. (2007). The relationship of physical fitness and motor competence to physical activity. Journal of Teaching in Physical Education, 26, 358-374.

Cole, T., Bellizzi, M., Flegal, K., \& Dietz, W. (2000). Establishing a standard definition for child overweight and obesity worldwide:
International survey. British Medical Journal, 320, $1-6$.

D'Hondt, E., Deforche, B., Bourdeaudhuij, I., \& Lenoir, M. (2009). Relationship between motor skill and body mass index in 5- to 10-year-old children. Adapted Physical Activity Quarterly, 26, 21-37.

Dencker, M., Thorsson, O., Karlsson, M., Lindén, C., Svensson, J., Wollmer, P., et al. (2006). Daily physical activity and its relation to aerobic fitness in children aged 8-11 years. European Journal of Applied Physiology, 96, 587592.

Fisher, A., Reilly, J., Kelly, L., Montgomery, C., Williamson, A., Paton, J., et al. (2004). Fundamental movement skills and habitual physical activity in young children. Medicine $\mathcal{E}$ Science in Sports \& Exercise, 37, 684-688.

Graf, C., Koch, B., Kretschamann-Kandel, E., Falkowski, G., Christ, H., Corburger, S., et al. (2004). Correlation between BMI, leisure habits and motor abilities in childhood (CHILT-Project). International Journal of Obesity, 28, 22-26.

Grund, A., Dilba, B., Forberger, K., Krause, H., Siewers, M., Rieckert, H., et al. (2000). Relationships between physical activity, physical fitness, muscle strenght and nutritional state in 5- to 11-year-old children. European Journal of Applied Physiology, 82, 425438.

Haga, M. (2008). The relationship between physical fitness and motor competence in children. Child: Care, Health and Development, 34, 329-334.

Hands, B., Larkin, D., Parker, H., Straker, L., \& Perry, M. (2009). The relationship among physical activity, motor competence and health-related fitness in 14-year-old adolescents. Scandinavian Journal of Medicine and Science in Sports, 19, 655-663.

Houwen, S., Hartman, E., \& Visscher, C. (2008). Physical activity and motor skills in children with and without visual impairments. Medicine \& Science in Sports \& Exercise, 41, 103-109.

Hume, C., Okely, A., Bagley, S., Telford, A., Booth, M., Crawford, D., et al. (2008). Does weight status influence associations between children's fundamental movement skills and physical activity? Research Quarterly for Exercise and Sport, 79, 158-165.

Janz, K., Dawson, J., \& Mahoney, L. (2000). Tracking physical fitness and physical activity 
from childhood to adolescence: The Muscatine study. Medicine \& Science in Sports \& Exercise, 32, 1250-1257.

Kemper, H., Vente, W., Mechelen, W., \& Twisk, J. (2001). Adolescent motor skills and performance: Is physical activity in adolescence related to adult physical fitness? American Journal of Human Biology, 13, 180-189.

Lazaar, N., Aucouturier, J., Ratel, S., Rance, M., Meyer, M., \& Duché, P. (2007). Effect of physical activity intervention on body composition in young children: Influence of body mass index status and gender. Acta Pediatrica, 96, 1315-1320.

Malina, R. (2001). Physical activity and fitness: Pathways from childhood to adulthood. American Journal of Human Biology, 13, 162-172.

McDowell, M., Fryar, C., Hirsch, R., \& Ogden, C. (2005). Anthropometric reference data for children and adults: U.S. population, 1999-2002 - Advance data from vital and health statistics, 361. Hyattsville, MD: National Centre for Health Statistics.

Minck, M., Ruiter, L., Van Mechelen, W., Kemper, H., \& Twisk, J. (2000). Physical fitness, body fatness, and physical activity: The Amsterdam Growth and Health Study. American Journal of Human Biology, 12, 593-599.

Mota, J., Flores, L., Flores, L. Ribeiro, J., \& Santos, M. (2006). Relationship of single measures of cardiorespiratory fitness and obesity in young schoolchildren. American Journal of Human Biology, 18, 335-341.

Oja, P. (2001). Dose response between total volume of physical activity and health and fitness. Medicine $\mathcal{E}$ Science in Sports $\mathcal{E}$ Exercise, 33(6), S428-S437.

Okely, A., Booth, M., \& Patterson, J. (2001). Relationship of physical activity to fundamental movement skills among adolescents. Medicine $\mathcal{E}$ Science in Sports \& Exercise, 33(11), 1899-1904.

Padez, C., Fernandes, T., Mourão, I., Moreira, P., \& Rosado, V. (2004). Prevalence of overweight and obesity 7-9-year-old Portuguese children: Trends in body mass index from 1970-2002. American Journal of Human Biology, 16, 670-678.

Raudsepp, L., \& Päll, P. (2006). The relationship between fundamental motor skills and outside school physical activity of elementary school children. Pediatric Exercise Science, 18, 426-435.

Reed, J. A., \& Metzker, A. (2004). Relationships between physical activity and motor skills in middle school children. Perceptual and Motor Skills, 99, 483-494.

Reiff, G., Dixon, W., Jacoby, D., Ye, G., Spain, C., \& Huniscker, P. (1986). The President's Council on physical fitness and sports national school population fitness survey. Ann Arbor, MI: University of Michigan.

Rodrigues, L., Sá, C., Bezerra, P., \& Saraiva, L. (2006). Estudo morfofuncional da criança Vianense. Viana do Castelo: Câmara Municipal de Viana do Castelo.

Rose, B., Larkin, D., \& Berger, B. (1998). The importance of motor coordination for children's motivational orientations in sport. Adapted Physical Activity Quarterly, 15, 316-327.

Sallis, J., Prochaska, J., \& Taylor, W. (2000). A review of correlates of physical activity of children and adolescents. Medicine $\mathcal{E}$ Science in Sports \& Exercise, 32 (5), 963-975.

Simon, J., Beunen, G., Renson, R., Claessens, A., Vanreusel, B., \& Lefevre, J. (1990). Growth and fitness of Flemish girls: The Leuven Growth Study. Champaign, IL: Human Kinetics Publishers.

Stodden, D., Goodway, J., Langendorfer, S., Roberton, M., Rudisill, M., Garcia, C., et al. (2008). A developmental perspective on the role of motor skill competence in physical activity: An emergent relationship. Quest, 60, 290-306.

Stratton, G., Canoy, D., Boddy, L., Taylor, S., Hackett, A., \& Buchan, I. (2007). Cardiorespiratory fitness and body mass index of 9-11-year-old English children: A serial cross-sectional study from 1998 to 2004. International Journal of Obesity, 31, 1172-1178.

Willms, J. (2003). Ten hypotheses about socioeconomic gradients comes. Otawa, Canada: Human Resources Development of Canada.

Wrotniak, B., Epstein, L., Dorn, J., Jones, K., \& Kondilis, V. (2006). The relationship between motor proficiency and physical activity in children. Pediatrics, 118, e1758-e1765. 